\title{
The use of cell free DNA in the diagnosis of HCC
}

\author{
Bubu A. Banini, Arun J. Sanyal \\ Division of Gastroenterology, Hepatology and Nutrition, Department of Internal Medicine, Virginia Commonwealth University, \\ Richmond, VA 23298, USA.
}

Correspondence to: Dr. Arun J. Sanyal and Dr. Bubu A. Banini, Division of Gastroenterology, Hepatology and Nutrition, Department of Internal Medicine, Virginia Commonwealth University, MCV Box 980341, Richmond, VA 23298, USA. Email: arun.sanyal@vcuhealth.org; bubu.banini@vcuhealth.org

How to cite this article: Banini BA, Sanyal AJ. The use of cell free DNA in the diagnosis of HCC. Hepatoma Res 2019;5:34. http://dx.doi.org/10.20517/2394-5079.2019.30

Received: 1 Aug 2019 First Decision: 15 Aug 2019 Revised: 22 Aug 2019 Accepted: 29 Aug 2019 Published: 23 Sep 2019

Science Editor: Jin-Lin Hou Copy Editor: Cai-Hong Wang Production Editor: Jing Yu

\begin{abstract}
Hepatocellular carcinoma (HCC) is one of the most common malignant tumors worldwide and is associated with high mortality. The currently used methods for diagnosing HCC, including imaging modalities and liver biopsy, detect tumors at a relatively advanced stage or are invasive. Non-invasive biomarkers are urgently needed to facilitate screening and early diagnosis of HCC, as well as treatment monitoring and detection of tumor recurrence. Liquid biopsy, the analysis of blood or other body fluids to obtain genetic and epigenetic information, has historically been applied to other types of cancer including breast and prostate cancer. Over the past few decades, liquid biopsy analysis has shed significant insights on genetic and epigenetic aberrations in HCC detectable in peripheral blood. Aberrations in nucleic acids found circulating freely in body fluids or contained within extracellular vesicles such as exosomes or microvesicles show potential clinical utility as non-invasive biomarkers. In this review, we present available literature on cell-free nucleic acids in the diagnosis of HCC.
\end{abstract}

Keywords: Hepatocellular carcinoma, liquid biopsy, cell free nucleic acid, cell free DNA, exosomes, microvesicles, biomarkers

\section{INTRODUCTION}

Hepatocellular cancer (HCC) has become the second leading cause of cancer deaths worldwide ${ }^{[1]}$. Unfortunately, most cases of HCC are undetected until late stage due to absence of symptoms in early stage HCC, and the lack of sensitive and convenient methods of screening. Previous estimates showed that the

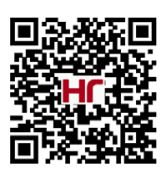


one-year survival for HCC in the United States is less than $50 \%$, while the five-year survival is $10 \%{ }^{[2]}$. With the advent of potent therapy for chronic hepatitis $\mathrm{C}$ virus (HCV) infection, the overall global incidence of HCC may plateau or decrease as a result of decreased HCV-associated HCC, however these gains appear to be threatened by the increasing incidence of nonalcoholic fatty liver disease (NAFLD)-associated HCC and the persistently high levels of hepatitis B virus (HBV)-associated HCC.

Diagnosis of HCC can be made by imaging studies such as multiphasic computed tomography scan or magnetic resonance imaging. However, tissue biopsy remains the gold-standard for HCC diagnosis especially in non-cirrhotic patients or those with nonspecific imaging studies. The risks of biopsy include procedure-related complications such as pain, bleeding, and perforation of adjacent organs, as well as tumor seeding along the needle track and sampling errors resulting in false negative results. Aside from these risks, technological advancements over the past few decades have led to a better understanding of the high heterogeneity and dynamic evolution of HCC tumor cells, and a time- and location-constrained tissue biopsy is inadequate in reflecting these dynamic changes. These shortcomings have led to increased interest in the application of liquid biopsy analysis to HCC. Although the concept of liquid biopsy has been in existence for several decades, the term gained traction in the early 2000's, with one of its first uses pertaining to the capture of circulating tumor cells (CTCs) for biomarker analysis in breast cancer patients $^{[3]}$.

Liquid biopsy generally refers to the analysis of blood or other body fluids to obtain genetic or epigenetic information which can be applied in screening, diagnosis, prognostication, treatment monitoring or disease recurrence $^{[4]}$. The major advantage of liquid biopsy is non-invasiveness which makes it attractive for frequent analysis to track mutations and other molecular changes over time. The most commonly used HCC serum biomarker is serum alpha-fetoprotein (AFP), together with its fucosylated glycoform (AFP-L3). AFP is normally produced during gestation by the fetal liver and yolk sac, and levels decline rapidly after birth. Regeneration of liver cells leads to AFP production, as can be seen in chronic liver disease and in HCC. Other types of malignancy, for instance testicular or ovarian cancer, can also cause AFP elevation. The AFP-L3 glycoform, named for its ability to bind Lens culinaris agglutinin, is a relatively new test developed in 1992 that is more specific for HCC, compared to $\mathrm{AFP}^{[5]}$. Serum AFP concentration can be normal even in advanced $\mathrm{HCC}^{[6]}$. In two studies of approximately 1800 patients, AFP was found to have about $60 \%$ sensitivity and $80 \%$ specificity in detecting HCC using a cut off level between 10 to $20 \mathrm{ng} / \mathrm{mL}^{[7,8]}$. Higher serum AFP levels are associated with greater specificity and less sensitivity, for instance AFP $>400 \mathrm{ng} / \mathrm{mL}$ implies HCC until proven otherwise. However, fewer than $20 \%$ of HCC cases have such elevated AFP levels ${ }^{[9]}$.

Serum and plasma biomarkers detectable through liquid biopsy show promise in the early detection of HCC either alone or in combination with AFP. These markers have the potential to be adjunctive or superior to conventional methods of HCC diagnosis. Several of these markers, however, are still in preclinical development and testing and none of them has of yet been recommended for HCC diagnosis. Here, we provide an updated summary of cell-free nucleic acid (cfNA) analysis in the diagnosis HCC, with emphasis on cell-free DNA (cfDNA).

\section{LIQUID BIOPSY FOR HCC}

Liquid biopsy specimens contain genetic information in CTCs or in the form of cfNAs released by apoptotic cells or living cells. cfNA can be found circulating freely in body fluids or are taken up by extracellular vesicles such as exosomes and microvesicles. The various types of cfNA include cfDNA, mRNA (cfRNA), and noncoding RNAs including miRNAs (cfmiRNA). Other noncoding RNAs including long noncoding RNA, small nuclear RNA, small nucleolar RNA, and piwi-interacting RNA may also be present in liquid biopsy specimens and could potentially serve as biomarkers although there are currently very few studies on these subtypes. 
The first report of cfNA derived from human peripheral blood was published by Mandel and Metais ${ }^{[10]}$ in 1948, however its significance was not realized until several decades later in 1977 when it was discovered that serum and plasma from cancer patients carry higher concentrations of cfDNA compared to healthy individuals $^{[11]}$. About a decade later, Vasioukhin showed that cfDNA can have cancer characteristics, suggesting that cancer cells can release DNA into peripheral blood ${ }^{[12]}$. This notion was soon confirmed by other investigators ${ }^{[13,14]}$, and cfDNA released by cancer cells into circulation has been subsequently referred to as circulating tumor DNA (ctDNA).

Analysis of plasma and serum cfRNA is limited by the very small quantities present in circulation as well as degradation by ribonuclease (RNase). Incorporation of cfRNA into extracellular vesicles protects them from degradation. Over the past decade, several groups have shown that cfRNA can potentially be applied in HCC detection and monitoring ${ }^{[15-17]}$. A recent study by Xu et al. ${ }^{[18]}$ showed that serum mRNA levels of exosomal hnRNPH1 in patients with primarily HBV-associated HCC were significantly higher than in patients with chronic hepatitis B, liver cirrhosis, or healthy control ${ }^{[18]}$. Exosomal hnRNPH1 levels also associated with TNM stage, Child-Pugh classification, portal vein embolism and lymph node metastasis.

Non-coding RNA, especially cfmiRNAs were first demonstrated as a promising biomarker in patients with solid cancers in $2008^{[19]}$. Since then, there have been several studies on non-coding RNAs in different types of cancer including $\mathrm{HCC}^{[20]}$. A recent article mapped the differential expression of non-coding RNAs in normal liver tissue and in various stages of liver disease leading to HCC; each liver phenotype was found to demonstrate a unique RNA signature ${ }^{[21]}$. Induction of exosomal miR-21 and miR-10b in HCC was found to promote cancer cell proliferation and metastasis, potentially serving in prognostication and therapy for HCC $^{[22]}$. Several other cfmiRNAs have been studied, including miR-1 ${ }^{[23]}$, miR-16 ${ }^{[24-26]}$, and miR-122 ${ }^{[23,27-29]}$. An in-depth review of circulating miRNA signatures in HCC is beyond the scope of this article, and the reader is referred to a recent article by Mirzaei et al ${ }^{[30]}$ for further information.

Liquid biopsy analysis in HCC has significantly expanded over the past decade, providing substantial information on different HCC tumors and their microenvironment, and the potential application of such information to disease diagnosis and monitoring.

\section{Isolation of cell-free DNA in liquid biopsy samples}

There are several challenges in the isolation of cfDNA in general and ctDNA in particular, including DNA lysis as a results of blood clotting in collection tubes, DNA contamination during processing or DNA loss during isolation. Thus, the right sample collection tube and optimal processing methods are crucial to the success of isolation and to the accuracy of the sample obtained. A $1 \mathrm{~mL}$ volume of blood typically yields $10 \mathrm{ng}$ of cfDNA, and in cancer patients, about $0.01 \%$ to $1 \%$ of cfDNA comprises ctDNA. Several methods have been used in the isolation of ctDNA, including targeted methods involving polymerase chain reaction (PCR) based on known genetic mutations, for instance digital PCR; bead, emulsion, amplification and magnetics (BEAMing) PCR; and amplification-refractory mutation system-PCR. Alternatively, a variety of untargeted methods can be employed to sequence millions of DNA fragments, including Sanger sequencing and next-generation sequencing techniques such as targeted amplification sequencing or targeted capture sequencing ${ }^{[31,32]}$.

\section{HCC-ASSOCIATED QUANTITATIVE CHANGES IN CELL-FREE DNA}

Cancer is associated with both quantitative and qualitative changes in cfDNA detectable by liquid biopsy $^{[33-37]}$ [Figure 1]. In patients with HCC, total cfDNA concentration is significantly higher than in those without $\mathrm{HCC}^{[33,34]}$. Although non-specific, cfDNA increase in association with HCC has potential utility in screening for HCC, as well as in monitoring of treatment response and in predicting HCC 


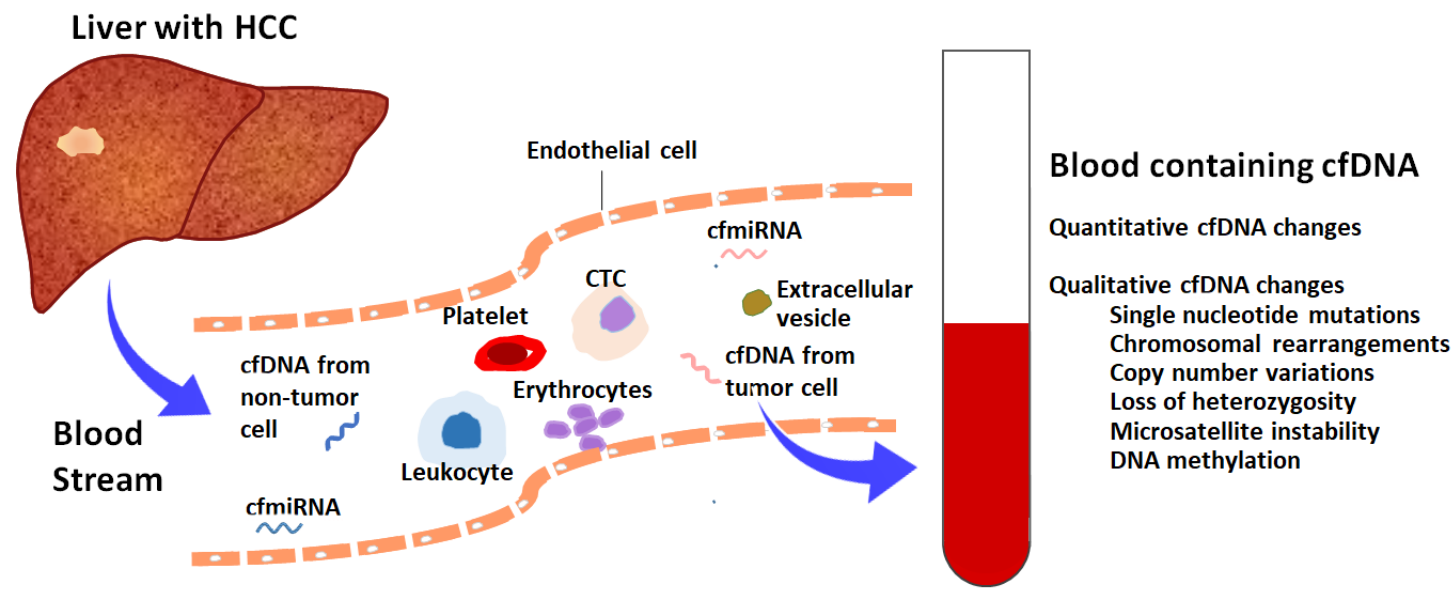

Figure 1. Liquid biopsy for hepatocellular carcinoma. Tumors release a number of molecules into circulation including tumor cells, cellfree DNA, different circulating RNA classes, proteins and extracellular vesicles including exosomes and microvesicles. Cell-free DNA can be isolated from blood or other body fluids and analyzed to determine genetic and epigenetic changes present in circulation which are reflective of changes occurring in tissues, potentially avoiding the need for invasive tissue sampling. cfDNA: cell free DNA; cfmiRNA: cell free microRNA; CTC: circulating tumor cell; HCC: hepatocellular carcinoma

recurrence ${ }^{[34,38-40]}$. A recent manuscript by Yan et al ${ }^{[41]}$ analyzed cfDNA and AFP levels from 24 patients with HCC and 62 patients with chronic hepatitis B with varying degrees of fibrosis (Fo to F6). Using multivariate analysis, the authors found that age and cfDNA were independent predictors of HCC, while AFP was not an independent predictor. They developed a combination model including cfDNA level, age and AFP, collectively referred to as HCC index, for HCC diagnosis by backward logistic regression analysis. The HCC index showed an area under the receiver operating characteristic curve (AUROC) of 0.98 (95\% confidence interval $0.92-1.00$ ), a sensitivity of $87 \%$ and specificity of $100 \%$ for the diagnosis of HCC at a cutoff value of $0.61^{[41]}$, proving superior to cfDNA alone or AFP alone in the diagnosis of $\mathrm{HCC}^{[41]}$. As shown by Yan et al. ${ }^{[41]}$, combination of cfDNA level with other protein or genetic biomarkers holds promise as a liquid-biopsy based clinical tool in the early diagnosis of HCC.

\section{HCC-ASSOCIATED QUALITATIVE CHANGES IN CELL-FREE DNA}

Genetic or epigenetic alterations in cfDNA in association with HCC are detectable by liquid biopsy and are reliable indicators of changes occurring in tumor tissues. In general, these changes are grouped into single nucleotide mutations ${ }^{[35,42]}$, variations in DNA copy number ${ }^{[35,36]}$, chromosomal rearrangements, loss of heterozygosity, microsatellite instability, and changes in methylation pattern ${ }^{[37]}$.

\section{Single nucleotide mutations}

Indepth genomic analysis of HCC tumor tissue to the base pair level has shown that no two tumors carry the same cadre of somatic mutations ${ }^{[43]}$. There is considerable variability in the number of mutations even among patients with advanced stage HCC, as demonstrated by analysis of three patients with advanced HCC which showed 7.2, 15 and 7,910 mutant fragments per $5 \mathrm{~mL}$ of plasma ${ }^{[43]}$. Tumor-specific somatic mutations in several genes have been identified in the peripheral blood of HCC patients, including TP53 ${ }^{[44]}$, $H C K^{[45]}$ and TERT ${ }^{[46]}$.

The three most frequent somatic mutations in HCC are TERT promoter activating mutations which are found in 40\%-60\% of HCC patients; and the mutually exclusive TP53 and CTNNB1 mutations which are found in $30-50 \%$ of HCC cases $^{[47-49]}$. Digital droplet PCR to interrogate the single nucleotide mutations TERT c.-124C>T, TP53 c.747G>T (p.R249S), CTNNB1 c.121A>G (p.T41A) and c.133T>C (p.S45A) in the 
peripheral blood of patients with predominantly HBV-positive and BCLC stage A showed that 56\% of the patients harbored ctDNA containing these mutations ${ }^{[46]}$. TERT promoter mutations, however, have been observed in both HCC patients as well as non-HCC cirrhotic patients, suggesting limited utility as a biomarker for $\mathrm{HCC}^{[50-52]}$. On the other hand, the TP53 p.R249S mutation appears specific for HCC and has been identified in plasma, serum and urine samples obtained from cancer patients ${ }^{[33-57]}$. The TP53 p.R249S mutation is more common in HBV- and aflatoxin-associated HCC, compared to HCC associated with other etiologies. ctDNA in 14 patients with advanced HCC using next generation sequencing showed that somatic CTNNB1 mutations were the second most common mutation and occurred in $29 \%$ of the patients studied ${ }^{[58]}$.

The significant heterogeneity of HCC genetics in association with different etiologies (for instance alcohol related liver disease vs. HBV vs. HCV vs. NAFLD) has posed a major challenge to the development of a universal biomarker panel for detecting HCC. This challenge necessitates the integration of multiple genes and multiple loci within a given gene, as well as combining a vast array of protein and genetic biomarkers. CancerSEEK is a recently developed blood test which detects eight tumor-associated protein biomarkers and mutations (including single base substitutions) in 1933 distinct genomic positions ${ }^{[59]}$. The test was used to query peripheral blood derived from 812 healthy controls and 192 non-metastatic cancers of the breast, colorectum, esophagus, liver, lung, ovary, pancreas and stomach ${ }^{[59]}$. Among 44 patients with HCC, the test a showed $98 \%$ sensitivity and $99 \%$ specificity in cancer detection. Overall, the test detected five cancer types with sensitivities ranging from $69 \%$ to $98 \%$, and with over $99 \%$ specificity. The performance of CancerSEEK in differentiating HCC patients from other high risk patients, for instance those with advanced fibrosis or cirrhosis, is yet to be studied.

\section{Chromosomal rearrangements}

Genomic sequencing has identified a number of chromosomal rearrangements in HCC. Ono et al. ${ }^{[0]}$ determined cancer-associated genomic rearrangements in HCC tumors through whole-genome sequencing. Subsequently, they validated some of these rearrangements by means of PCR using ctDNA isolated pre-operatively from peripheral blood of HCC patients and primers designed to detect the breakpoints of chromosomal rearrangements seen in tumor tissue. The authors found that pre-operative ctDNA from 7 HCC patients showed several deletions, inversions, tandem duplications and translocations seen in HCC tumor tissue ${ }^{[60]}$. Chromosomal rearrangements can lead to copy number variations and other genetic aberrations, potentially serving as an early noninvasive marker for HCC.

\section{Copy number variations}

Shotgun massively parallel sequencing (MPS) was used to determine tumor-associated copy number variations in the tumor tissue of 4 HCC patients, and in their plasma pre- and post-resection of tumor, compared to 16 healthy controls ${ }^{[35]}$. Characteristic copy number variations in tumor tissue were reflected in pre-resection plasma samples, and were missing almost entirely in post-resection plasma samples. The pre-resection plasma samples detected approximately $10 \%$ to $100 \%$ of tumor-associated copy number aberrations seen in their corresponding tumor tissue, with detectability of plasma copy number aberrations strongly correlating with plasma ctDNA concentration ${ }^{[35]}$. In another study, MPS analysis of plasma ctDNA size in 90 HCC patients compared to patients with chronic hepatitis $\mathrm{B}(n=67)$, hepatitis B-associated cirrhosis $(n=36)$, and healthy controls $(n=32)$ showed that HCC plasma carried high levels of aberrantly short and long DNA ${ }^{[36]}$. The short ctDNA preferentially carried tumor-associated copy number aberrations. Among the 90 HCC patients, 76 (84\%) had at least one chromosomal arm-level copy number aberration on chromosomes 1 or 8 . In addition, plasma derived from HCC patients contained high levels of mitochondrial DNA albeit much shorter than nuclear DNA ${ }^{[36]}$. The observation that cfDNA in HCC patients are shorter and more fragmented than in patients without liver disease or with nonmalignant liver processes has been made by several other investigators ${ }^{[36,61]}$. This observation is worthy of 
further investigation and may have clinical utility in the diagnosis or monitoring of HCC either alone or in combination with other biomarkers.

\section{Loss of heterozygosity and microsatellite instability}

Pang et al. ${ }^{[62]}$ used three high-polymorphic microsatellite markers located on chromosome 8p, D8S277, D8S298 and D8S1771 to examine loss of heterozygosity ( $\mathrm{LOH}$ ) and microsatellite instability. By analyzing plasma cfDNA and tumor tissues from 62 HCC patients, they examined the features of these aberrations in peripheral blood and determined their concordance with tumor tissue. $\mathrm{LOH}$ in one or more of the three examined loci was identified in about $58 \%$ of patients, occurring at a higher rate in those with metastatic HCC (63\%) compared to those with non-metastatic disease $(26 \%)^{[62]}$. Majority of patients carried microsatellite instability in plasma samples at the same loci as their corresponding HCC tissues, with a concordance rate of about $73 \%{ }^{[63]}$. Their findings suggest that $\mathrm{LOH}$ and microsatellite alterations may potentially serve in non-invasive diagnosis of HCC, however these alterations generally occur less commonly than the other genetic alterations discussed, and studies are needed to delineate the clinical applicability of these observations.

\section{Alterations in DNA methylation}

DNA methylation, one of the earliest known and well-studied epigenetic modifications, confers changes in chromatin structure, DNA stability and DNA-protein interactions to modify gene expression. Methylation events occur very early in carcinogenesis hence are often detected in precancerous states. To date, several studies have showed that altered DNA methylation at several genes are associated with the initiation and progression of HCC, including $p 15$ and $p 16^{[64]}, A P C^{[65]}, S P I N T 2^{[66]}, S F R P 1^{[67]}, T F P 12^{[68]}, G S T P 1^{[69]}$ and $R A S S F 1 A^{[70]}$. NAFLD-related HCC is associated with hypermethylation of the glycine N-methyltransferase $(G N M T)$ promoter, resulting in reduced gene expression ${ }^{[7]}$. Differential DNA hypomethylation has also been seen in HCC. DNA hypomethylation is known to induce several processes leading to transposon activation, chromosomal instability, and the generation of copy number variations. Hypomethylation of repetitive DNA sequences by way of long interspersed nucleotide elements 1 (LINE-1) has been detected in the plasma of patients with $\mathrm{HCC}^{[72]}$. Concordance in the methylation profile of several tumor suppressor genes between HCC plasma and tumor tissue has been demonstrated by several studies.

Wong et al. ${ }^{[37]}$ showed that $25 \%$ of patients with $p 15$ methylation in tissue also demonstrated methylated p15 in blood samples, and nearly all patients with $p 15$ and $p 16$ methylation in tissues demonstrated methylation abnormalities in blood samples. Importantly, patients with p15 and p16 methylation developed HCC metastasis or recurrence after treatment, suggesting that analysis of p15/p16 methylation in cfDNA derived from peripheral blood can serve as a biomarker for predicting the metastasis or recurrence of HCC.

Iyer et al ${ }^{[65]}$ analyzed the tumor methylation profile of several tumor suppressor genes including APC, FHIT and E-cadherin through analysis of plasma and corresponding tumor DNA from 28 HCC patients, as well as plasma DNA from age and sex-matched controls. The analysis showed a statistically significant concordance in methylation profile between plasma and corresponding tumor DNA for all genes analyzed. The concordance for APC methylation in plasma DNA vs. HCC tumor tissue was almost $82 \%$, with sensitivity and specificity of $78 \%$ and $90 \%$. For FHIT, the concordance, sensitivity and specificity were all approximately $86 \%$. For E-cadherin, concordance was $79 \%$, with sensitivity and specificity of $68 \%$ and $100 \%$. As in other studies, $p 15$ and $p 16$ methylation patterns were also found to be concordant with sensitivities ranging from $50 \%-60 \%$ and specificities in the $85 \%-95 \%$ range.

RASSF1A, a member of the Ras association domain family protein is a tumor suppressor frequently silenced in malignancy by hypermethylation. Serum analysis showed that $90 \%$ of HCC patients and $62.5 \%$ of HCV patients demonstrate RASSF1A hypermethylation, compared to $10 \%$ in healthy serum ${ }^{[73]}$. 
A biomarker panel based on analysis of a number of genes may serve to better differentiate HCC blood from normal samples, as shown for a combined analysis of the methylation pattern of four genes APC, GSTP1, RASSF1 A, and SFRP1 which showed an AUCROC of 0.933 in identifying HCC from normal samples, compared to 0.800 to 0.881 for the individual genes ${ }^{[67]}$. In another study to evaluate the potential of ctDNA methylation patterns in the diagnosis and prognostication of HCC, Xu et al ${ }^{[7]}$ identified a methylation marker panel differentially enriched in HCC tissue compared to blood leukocytes of healthy individuals. In a training data set of 715 HCC samples and 560 normal samples, the sensitivity and specificity of a 10-marker panel based on methylation patterns were $85.7 \%$ and $94.3 \%$, respectively, and a combined prognostic score based on these markers significantly correlated with risk of death ${ }^{[7]}$. These studies suggest that methylation changes characteristic of HCC can be reliably identified in peripheral blood samples and potentially serve as biomarkers for diagnosis and prognostication of HCC.

\section{CONCLUSION}

Liquid biopsy analysis of serum and plasma can reliably detect genetic and epigenetic alterations present in HCC tumor tissue, providing a less invasive alternative to the current gold standard of liver biopsy. Due to the significant heterogeneity of HCC, a single biomarker would lack the requisite sensitivity and specificity for HCC diagnosis, hence a panel consisting of multiple genetic and epigenetic alterations, likely in combination with protein biomarkers, would have the best diagnostic utility. One such test is CancerSEEK, which detects eight tumor-associated protein biomarkers and mutations in 1933 distinct genomic positions, with $98 \%$ sensitivity and $99 \%$ specificity for HCC detection when tested in 44 HCC patients and 812 controls $^{[59]}$. The authors estimated a cost of about $\$ 500$ to perform a CancerSEEK analysis ${ }^{[59]}$. Although the test holds promise for diagnosing and monitoring HCC, further studies, including performance of the assay in patients at high risk for HCC such as those with advanced fibrosis or cirrhosis would need to be undertaken. Several other analyses of cfDNA biomarkers either alone or in combination with non-nucleic acid biomarkers for non-invasive diagnosis of HCC are in progress.

\section{DECLARATIONS}

\section{Authors' contributions}

Study concept and design: Banini BA, Sanyal AJ

Literature search: Banini BA

Drafting of the manuscript: Banini BA

Critical revision of the manuscript for important intellectual content: Banini BA, Sanyal AJ

\section{Availability of data and materials}

Not applicable.

\section{Financial support and sponsorship}

This work was supported by the NIH Grants (5T32 DK07150, RO1 DK 10596).

\section{Conflicts of interest}

Both authors declared that there are no conflicts of interest. Dr. Sanyal is President of Sanyal Biotechnology and has stock options in Tiziana, Durect, Indalo, Inversago. He has served as a consultant to Medimmune, Astra Zeneca, Nitto Denko, Nimbus, Salix, Tobira, Takeda, Terns, Conatus, Lilly, Poxel, Blade, Surrozen, Birdrock, Siemens, Madrigal, Novartis, Pfizer, Hemoshear, Novo Nordisk, Gilead, Exhalenz, Bristol Myers Squibb, Glympse and Genfit. He has been an unpaid consultant to Intercept, Zafgen, Prosciento, Iquvia, NGM Bio, Echosens, Immuron, Syntlogic, Zafgen, Zydus, Nordic Bioscience. His institution has received grant support from Gilead, Salix, Tobira, Intercept, Merck, Astra Zeneca, Zydus and Novartis. 


\section{Ethical approval and consent to participate}

Not applicable.

\section{Consent for publication}

Not applicable.

\section{Copyright}

(c) The Author(s) 2019.

\section{REFERENCES}

1. Banini BA, Roberts LR. Hepatocellular carcinoma. Complications of Cirrhosis: Evaluation and management. Springer International Publishing; 2015. pp. 1-370.

2. Altekruse SF, McGlynn KA, Reichman ME. Hepatocellular carcinoma incidence, mortality, and survival trends in the United States from 1975 to 2005. J Clin Oncol 2009;27:1485-91.

3. Punnoose EA, Atwal SK, Spoerke JM, Savage H, Pandita A, et al. Molecular biomarker analyses using circulating tumor cells. PLoS One 2010;5:e12517.

4. Crowley E, Di Nicolantonio F, Loupakis F, Bardelli A. Liquid biopsy: monitoring cancer-genetics in the blood. Nat Rev Clin Oncol 2013;10:472-84.

5. Hirai H, Taketa K. Lectin affinity electrophoresis of alpha-fetoprotein. Increased specificity and sensitivity as a marker of hepatocellular carcinoma. J Chromatogr 1992;604:91-4.

6. Bruix J, Sherman M, American Association for the Study of Liver D. Management of hepatocellular carcinoma: an update. Hepatology 2011;53:1020-2.

7. Marrero JA, Feng Z, Wang Y, Nguyen MH, Befeler AS, et al. Alpha-fetoprotein, des-gamma carboxyprothrombin, and lectin-bound alpha-fetoprotein in early hepatocellular carcinoma. Gastroenterology 2009;137:110-8.

8. Lok AS, Sterling RK, Everhart JE, Wright EC, Hoefs JC, et al. Des-gamma-carboxy prothrombin and alpha-fetoprotein as biomarkers for the early detection of hepatocellular carcinoma. Gastroenterology 2010;138:493-502.

9. Farinati F, Marino D, De Giorgio M, Baldan A, Cantarini M, et al. Diagnostic and prognostic role of alpha-fetoprotein in hepatocellular carcinoma: both or neither? Am J Gastroenterol 2006;101:524-32.

10. Mandel P, Metais P. Les acides nucléiques du plasma sanguin chez l'homme. C R Seances Soc Biol Fil 1948;142:241-3.

11. Leon SA, Shapiro B, Sklaroff DM, Yaros MJ. Free DNA in the serum of cancer patients and the effect of therapy. Cancer Res 1977;37:646-50.

12. Vasioukhin V, Anker P, Maurice P, Lyautey J, Lederrey C, et al. Point mutations of the N-ras gene in the blood plasma DNA of patients with myelodysplastic syndrome or acute myelogenous leukaemia. Br J Haematol 1994;86:774-9.

13. Cachia PG, Taylor C, Thompson PW, Tennant GB, Masters G, et al. Non-dysplastic myelodysplasia? Leukemia 1994;8:677-81.

14. Sorenson GD, Pribish DM, Valone FH, Memoli VA, Bzik DJ, et al. Soluble normal and mutated DNA sequences from single-copy genes in human blood. Cancer Epidemiol Biomarkers Prev 1994;3:67-71.

15. Matsumura M, Shiratori Y, Niwa Y, Tanaka T, Ogura K, et al. Presence of alpha-fetoprotein mRNA in blood correlates with outcome in patients with hepatocellular carcinoma. J Hepatol 1999;31:332-9.

16. Cillo U, Navaglia F, Vitale A, Molari A, Basso D, et al. Clinical significance of alpha-fetoprotein mRNA in blood of patients with hepatocellular carcinoma. Clin Chim Acta 2004;347:129-38.

17. Jeng KS, Sheen IS, Tsai YC. Does the presence of circulating hepatocellular carcinoma cells indicate a risk of recurrence after resection? Am J Gastroenterol 2004;99:1503-9.

18. Xu H, Dong X, Chen Y, Wang X. Serum exosomal hnRNPH1 mRNA as a novel marker for hepatocellular carcinoma. Clin Chem Lab Med 2018;56:479-84.

19. Mitchell PS, Parkin RK, Kroh EM, Fritz BR, Wyman SK, et al. Circulating microRNAs as stable blood-based markers for cancer detection. Proc Natl Acad Sci U S A 2008;105:10513-8.

20. Zheng Q, Zhao J, Yu H, Zong H, He X, et al. Tumor-specific transcripts are frequently expressed in hepatocellular carcinoma with clinical implication and potential function. Hepatology 2019; doi: 10.1002/hep.30805.

21. Koduru SV, Leberfinger AN, Kawasawa YI, Mahajan M, Gusani NJ, et al. Non-coding RNAs in various stages of liver disease leading to hepatocellular carcinoma: differential expression of miRNAs, piRNAs, lncRNAs, circRNAs, and sno/mt-RNAs. Sci Rep 2018;8:7967.

22. Tian XP, Wang CY, Jin XH, Li M, Wang FW, et al. Acidic microenvironment up-regulates exosomal miR-21 and miR-10b in earlystage hepatocellular carcinoma to promote cancer cell proliferation and metastasis. Theranostics 2019;9:1965-79.

23. Koberle V, Kronenberger B, Pleli T, Trojan J, Imelmann E, et al. Serum microRNA-1 and microRNA-122 are prognostic markers in patients with hepatocellular carcinoma. Eur J Cancer 2013;49:3442-9.

24. Qu KZ, Zhang K, Li H, Afdhal NH, Albitar M. Circulating microRNAs as biomarkers for hepatocellular carcinoma. J Clin Gastroenterol 2011;45:355-60. 
25. Ge W, Yu DC, Li QG, Chen X, Zhang CY, et al. Expression of serum miR-16, let-7f, and miR-21 in patients with hepatocellular carcinoma and their clinical significances. Clin Lab 2014;60:427-34.

26. El-Abd NE, Fawzy NA, El-Sheikh SM, Soliman ME. Circulating miRNA-122, miRNA-199a, and miRNA-16 as biomarkers for early detection of hepatocellular carcinoma in egyptian patients with chronic hepatitis C virus infection. Mol Diagn Ther 2015;19:213-20.

27. Qi P, Cheng SQ, Wang H, Li N, Chen YF, et al. Serum microRNAs as biomarkers for hepatocellular carcinoma in Chinese patients with chronic hepatitis B virus infection. PLoS One 2011;6:e28486.

28. Liu M, Liu J, Wang L, Wu H, Zhou C, et al. Association of serum microRNA expression in hepatocellular carcinomas treated with transarterial chemoembolization and patient survival. PLoS One 2014;9:e109347.

29. Zekri AN, Youssef AS, El-Desouky ED, Ahmed OS, Lotfy MM, et al. Serum microRNA panels as potential biomarkers for early detection of hepatocellular carcinoma on top of HCV infection. Tumour Biol 2016;37:12273-86.

30. Mirzaei HR, Sahebkar A, Mohammadi M, Yari R, Salehi H, et al. Circulating microRNAs in hepatocellular carcinoma: potential diagnostic and prognostic biomarkers. Curr Pharm Des 2016;22:5257-69.

31. Forshew T, Murtaza M, Parkinson C, Gale D, Tsui DW, et al. Noninvasive identification and monitoring of cancer mutations by targeted deep sequencing of plasma DNA. Sci Transl Med 2012;4:136ra68.

32. Newman AM, Bratman SV, To J, Wynne JF, Eclov NC, et al. An ultrasensitive method for quantitating circulating tumor DNA with broad patient coverage. Nat Med 2014;20:548-54.

33. Chen H, Sun LY, Zheng HQ, Zhang QF, Jin XM. Total serum DNA and DNA integrity: diagnostic value in patients with hepatitis B virus-related hepatocellular carcinoma. Pathology 2012;44:318-24.

34. Huang Z, Hua D, Hu Y, Cheng Z, Zhou X, et al. Quantitation of plasma circulating DNA using quantitative PCR for the detection of hepatocellular carcinoma. Pathol Oncol Res 2012;18:271-6.

35. Chan KC, Jiang P, Zheng YW, Liao GJ, Sun H, et al. Cancer genome scanning in plasma: detection of tumor-associated copy number aberrations, single-nucleotide variants, and tumoral heterogeneity by massively parallel sequencing. Clin Chem 2013;59:211-24.

36. Jiang P, Chan $\mathrm{CW}$, Chan $\mathrm{KC}$, Cheng SH, Wong J, et al. Lengthening and shortening of plasma DNA in hepatocellular carcinoma patients. Proc Natl Acad Sci U S A 2015;112:E1317-25.

37. Wong IH, Lo YM, Yeo W, Lau WY, Johnson PJ. Frequent p15 promoter methylation in tumor and peripheral blood from hepatocellular carcinoma patients. Clin Cancer Res 2000;6:3516-21.

38. Ren N, Qin LX, Tu H, Liu YK, Zhang BH, et al. The prognostic value of circulating plasma DNA level and its allelic imbalance on chromosome 8p in patients with hepatocellular carcinoma. J Cancer Res Clin Oncol 2006;132:399-407.

39. Iizuka N, Sakaida I, Moribe T, Fujita N, Miura T, et al. Elevated levels of circulating cell-free DNA in the blood of patients with hepatitis C virus-associated hepatocellular carcinoma. Anticancer Res 2006;26:4713-9.

40. Piciocchi M, Cardin R, Vitale A, Vanin V, Giacomin A, et al. Circulating free DNA in the progression of liver damage to hepatocellular carcinoma. Hepatol Int 2013;7:1050-7.

41. Yan L, Chen Y, Zhou J, Zhao H, Zhang H, et al. Diagnostic value of circulating cell-free DNA levels for hepatocellular carcinoma. Int J Infect Dis 2018;67:92-7.

42. Szymanska K, Chen JG, Cui Y, Gong YY, Turner PC, et al. TP53 R249S mutations, exposure to aflatoxin, and occurrence of hepatocellular carcinoma in a cohort of chronic hepatitis B virus carriers from Qidong, China. Cancer Epidemiol Biomarkers Prev 2009;18:1638-43.

43. Bettegowda C, Sausen M, Leary RJ, Kinde I, Wang Y, et al. Detection of circulating tumor DNA in early- and late-stage human malignancies. Sci Transl Med 2014;6:224ra24.

44. Yu L, Liu X, Han C, Lu S, Zhu G, et al. XRCC1 rs25487 genetic variant and TP53 mutation at codon 249 predict clinical outcomes of hepatitis B virus-related hepatocellular carcinoma after hepatectomy: a cohort study for 10 years' follow up. Hepatol Res 2016;46:765-74.

45. Cai ZX, Chen G, Zeng YY, Dong XQ, Lin MJ, et al. Circulating tumor DNA profiling reveals clonal evolution and real-time disease progression in advanced hepatocellular carcinoma. Int J Cancer 2017;141:977-85.

46. Huang A, Zhang X, Zhou SL, Cao Y, Huang XW, et al. Detecting circulating tumor DNA in hepatocellular carcinoma patients using droplet digital PCR is feasible and reflects intratumoral heterogeneity. J Cancer 2016;7:1907-14.

47. Nault JC, Zucman-Rossi J. Genetics of hepatocellular carcinoma: the next generation. J Hepatol 2014;60:224-6.

48. Kan Z, Zheng H, Liu X, Li S, Barber TD, et al. Whole-genome sequencing identifies recurrent mutations in hepatocellular carcinoma. Genome Res 2013;23:1422-33.

49. Fujimoto A, Totoki Y, Abe T, Boroevich KA, Hosoda F, et al. Whole-genome sequencing of liver cancers identifies etiological influences on mutation patterns and recurrent mutations in chromatin regulators. Nat Genet 2012;44:760-4.

50. Nault JC, Mallet M, Pilati C, Calderaro J, Bioulac-Sage P, et al. High frequency of telomerase reverse-transcriptase promoter somatic mutations in hepatocellular carcinoma and preneoplastic lesions. Nat Commun 2013;4:2218.

51. Nault JC, Calderaro J, Di Tommaso L, Balabaud C, Zafrani ES, et al. Telomerase reverse transcriptase promoter mutation is an early somatic genetic alteration in the transformation of premalignant nodules in hepatocellular carcinoma on cirrhosis. Hepatology 2014;60:1983-92.

52. Pinyol R, Tovar V, Llovet JM. TERT promoter mutations: gatekeeper and driver of hepatocellular carcinoma. J Hepatol 2014;61:685-7.

53. Hann HW, Jain S, Park G, Steffen JD, Song W, et al. Detection of urine DNA markers for monitoring recurrent hepatocellular carcinoma. Hepatoma Res 2017;3:105-11.

54. Hosny G, Farahat N, Tayel H, Hainaut P. Ser-249 TP53 and CTNNB1 mutations in circulating free DNA of Egyptian patients with hepatocellular carcinoma versus chronic liver diseases. Cancer Lett 2008;264:201-8. 
55. Kuang SY, Lekawanvijit S, Maneekarn N, Thongsawat S, Brodovicz K, et al. Hepatitis B 1762T/1764A mutations, hepatitis C infection, and codon 249 p53 mutations in hepatocellular carcinomas from Thailand. Cancer Epidemiol Biomarkers Prev 2005;14:380-4.

56. Szymanska K, Lesi OA, Kirk GD, Sam O, Taniere P, et al. Ser-249TP53 mutation in tumour and plasma DNA of hepatocellular carcinoma patients from a high incidence area in the Gambia, West Africa. Int J Cancer 2004;110:374-9.

57. Lleonart ME, Kirk GD, Villar S, Lesi OA, Dasgupta A, et al. Quantitative analysis of plasma TP53 249Ser-mutated DNA by electrospray ionization mass spectrometry. Cancer Epidemiol Biomarkers Prev 2005;14:2956-62.

58. Ikeda S, Tsigelny IF, Skjevik AA, Kono Y, Mendler M, et al. Next-generation sequencing of circulating tumor DNA reveals frequent alterations in advanced hepatocellular carcinoma. Oncologist 2018;23:586-93.

59. Cohen JD, Li L, Wang Y, Thoburn C, Afsari B, et al. Detection and localization of surgically resectable cancers with a multi-analyte blood test. Science 2018;359:926-30.

60. Ono A, Fujimoto A, Yamamoto Y, Akamatsu S, Hiraga N, et al. Circulating tumor DNA analysis for liver cancers and its usefulness as a liquid biopsy. Cell Mol Gastroenterol Hepatol 2015;1:516-34.

61. Huang A, Zhang X, Zhou SL, Cao Y, Huang XW, et al. Plasma Circulating cell-free DNA integrity as a promising biomarker for diagnosis and surveillance in patients with hepatocellular carcinoma. J Cancer 2016;7:1798-803.

62. Pang JZ, Qin LX, Wang QQ, Ren N, Sun BS, et al. Loss of heterozygosity of plasma circulating DNA from hepatocellular carcinoma patients and its clinical significance. Zhonghua Gan Zang Bing Za Zhi 2007;15:906-9.

63. Pang JZ, Qin LX, Ren N, Ye QH, Ying WD, et al. Microsatellite alterations of circulating DNA in the plasma of patients with hepatocellular carcinoma. Zhonghua Yi Xue Za Zhi 2006;86:1662-5.

64. Zhang YJ, Wu HC, Shen J, Ahsan H, Tsai WY, et al. Predicting hepatocellular carcinoma by detection of aberrant promoter methylation in serum DNA. Clin Cancer Res 2007;13:2378-84.

65. Iyer P, Zekri AR, Hung CW, Schiefelbein E, Ismail K, et al. Concordance of DNA methylation pattern in plasma and tumor DNA of Egyptian hepatocellular carcinoma patients. Exp Mol Pathol 2010;88:107-11.

66. Iizuka N, Oka M, Sakaida I, Moribe T, Miura T, et al. Efficient detection of hepatocellular carcinoma by a hybrid blood test of epigenetic and classical protein markers. Clin Chim Acta 2011;412:152-8.

67. Huang ZH, Hu Y, Hua D, Wu YY, Song MX, et al. Quantitative analysis of multiple methylated genes in plasma for the diagnosis and prognosis of hepatocellular carcinoma. Exp Mol Pathol 2011;91:702-7.

68. Sun FK, Fan YC, Zhao J, Zhang F, Gao S, et al. Detection of TFPI2 methylation in the serum of hepatocellular carcinoma patients. Dig Dis Sci 2013;58:1010-5.

69. Chang H, Yi B, Li L, Zhang HY, Sun F, et al. Methylation of tumor associated genes in tissue and plasma samples from liver disease patients. Exp Mol Pathol 2008;85:96-100.

70. Yeo W, Wong N, Wong WL, Lai PB, Zhong S, et al. High frequency of promoter hypermethylation of RASSF1A in tumor and plasma of patients with hepatocellular carcinoma. Liver Int 2005;25:266-72.

71. Borowa-Mazgaj B, de Conti A, Tryndyak V, Steward CR, Jimenez L, et al. Gene expression and DNA methylation alterations in the glycine N-methyltransferase gene in diet-induced nonalcoholic fatty liver disease-associated carcinogenesis. Toxicol Sci 2019; doi: 10.1093/toxsci/kfz110.

72. Tangkijvanich P, Hourpai N, Rattanatanyong P, Wisedopas N, Mahachai V, et al. Serum LINE-1 hypomethylation as a potential prognostic marker for hepatocellular carcinoma. Clin Chim Acta 2007;379:127-33.

73. Mohamed NA, Swify EM, Amin NF, Soliman MM, Tag-Eldin LM, et al. Is serum level of methylated RASSF1A valuable in diagnosing hepatocellular carcinoma in patients with chronic viral hepatitis C? Arab J Gastroenterol 2012;13:111-5.

74. Xu RH, Wei W, Krawczyk M, Wang W, Luo H, et al. Circulating tumour DNA methylation markers for diagnosis and prognosis of hepatocellular carcinoma. Nat Mater 2017;16:1155-61. 